\title{
Polymyxins, the last-resort antibiotics: Mode of action, resistance emergence, and potential solutions
}

\author{
Saswat S Mohapatra* $\odot$, Sambit K Dwibedy and Indira Padhy \\ Molecular Microbiology Lab, Department of Bioscience and Bioinformatics, Khallikote University, \\ Konisi, Berhampur 761 008, Odisha, India \\ *Corresponding author (Email, saswatsmohapatra@gmail.com)
}

MS received 7 June 2021; accepted 3 August 2021

\begin{abstract}
Infections caused by multi-drug resistant (MDR) bacterial pathogens are a leading cause of mortality and morbidity across the world. Indiscriminate use of broad-spectrum antibiotics has seriously affected this situation. With the diminishing discovery of novel antibiotics, new treatment methods are urgently required to combat MDR pathogens. Polymyxins, the cationic lipopeptide antibiotics, discovered more than half a century ago, are considered to be the last-line of antibiotics available at the moment. This antibiotic shows a great bactericidal effect against Gram-negative bacteria. Polymyxins primarily target the bacterial membrane and disrupt them, causing lethality. Because of their membrane interacting mode of action, polymyxins cause nephrotoxicity and neurotoxicity in humans, limiting their usability. However, recent modifications in their chemical structure have been able to reduce the toxic effects. The development of better dosing regimens has also helped in getting better clinical outcomes in the infections caused by MDR pathogens. Since the mid1990s the use of polymyxins has increased manifold in clinical settings, resulting in the emergence of polymyxin-resistant strains. The risk posed by the polymyxin-resistant nosocomial pathogens such as the Enterobacteriaceae group, Pseudomonas aeruginosa, and Acinetobacter baumannii, etc. is very serious considering these pathogens are resistant to almost all available antibacterial drugs. In this review article, the mode of action of the polymyxins and the genetic regulatory mechanism responsible for the emergence of resistance are discussed. Specifically, this review aims to update our current understanding in the field and suggest possible solutions that can be pursued for future antibiotic development. As polymyxins primarily target the bacterial membranes, resistance to polymyxins arises primarily by the modification of the lipopolysaccharides (LPS) in the outer membrane $(\mathrm{OM})$. The LPS modification pathways are largely regulated by the bacterial two-component signal transduction (TCS) systems. Therefore, targeting or modulating the TCS signalling mechanisms can be pursued as an alternative to treat the infections caused by polymyxin-resistant MDR pathogens. In this review article, this aspect is also highlighted.
\end{abstract}

Keywords. LPS modification; PhoPQ; PmrAB; polymyxin; two-component system

\section{Introduction}

Infectious diseases caused by bacterial pathogens are one of the leading causes of death in developing nations; moreover, emerging and re-emerging bacterial pathogens are creating a substantial burden on the healthcare sector. The success of these emerging pathogens can be attributed to their survival in the host, and their increased adaptation and resistance to the available antibacterial drugs. The emergence of pathogens that are resistant to multiple antibiotics, including the last-resort ones, is increasingly being reported from several parts of the world. As per the current estimates, globally 700,000 people die annually due to infections caused by drug-resistant bacterial infections (Gandra et al. 2020; Limmathurotsakul et al. 2019). According to some estimates, by the year 2050, 10 million people would die annually because of the infections caused by drug-resistant pathogens, a significant number to this list would be contributed by the 
low- and middle-income countries (LMICs) (de Kraker et al. 2016; O'Neill 2016). This has far-reaching consequences not only in the healthcare sector but also on the global economy, because of the loss of manpower. Considering the unprecedented global threat posed by antimicrobial resistance (AMR), World Health Organization (WHO) in 2015, developed a Global Action Plan to tackle AMR (WHO 2015). An integral part of this framework the "One Health" approach takes into consideration- human health, which is largely influenced by that of the animals and the shared environment.

Though the global organizations and policymakers in different countries have recognized the threat of AMR and have taken several policy initiatives to create awareness, develop stewardship, increase funding in the research to tackle the AMR problem, the situation on the ground remains grim. The reason for such a situation is that the development of novel antibacterial drugs has not been able to keep pace with the emergence of novel drug-resistant pathogens. In the preceding three decades no new antibiotics have been developed. The low discovery rate of new drugs, and the rapid emergence of resistance to them, has often discouraged the pharmaceutical industries to invest in this area of research. Several reasons can be attributed to the rapid emergence of AMR in the clinical settings, the more common of which is the lack of quick diagnostic methods to determine the resistant pathogens in the primary health care facilities, often leading to the prescription of broad-spectrum antibiotics. This indiscriminate use of broad-spectrum antibiotics has reduced our arsenal to fight against novel drug-resistant pathogens. The current situation is such that most bacterial pathogens are resistant to the frontline drugs like carbapenems, imipenem, and vancomycin, etc. The ongoing global pandemic due to SARS-CoV-2 has also dramatically enhanced the use of several antibiotics to treat the secondary bacterial infections among the patients (Rawson et al. 2020a, b), the impact of which on the AMR will be required to be assessed in due course (Knight et al. 2021; Lai et al. 2021; Rawson et al. 2020a).

While novel antibiotics are increasingly being required to treat drug-resistant bacterial pathogens, the WHO has recommended a priority list of pathogens against which novel antibiotics are urgently required. This list includes Enterococcus faecium, Staphylococcus aureus, Klebsiella pneumoniae, Acinetobacter baumannii, Pseudomonas aeruginosa, and Enterobacter species (ESKAPE) (De Oliveira et al. 2020). While there is an urgent requirement of novel antibiotics against ESKAPE pathogens, MDR Mycobacterium tuberculosis, and other emerging pathogens, there is also a renewed interest in the polymyxins, a group of antibiotics that were discovered almost seventy years back and whose use had been discontinued considering their significant toxicity in the humans.

In this review article, we discuss the general mechanisms of antibiotic resistance with particular emphasis on our current understanding of the polymyxin group of antibiotics. Specifically, the mode of action of the polymyxins, the mechanism of resistance to them, and the possible methods to intervene in the polymyxin resistance development are highlighted. This article has been updated with information available in the literature till July 2021, concerning polymyxin mode of action and resistance mechanisms.

\section{Antimicrobial resistance}

Antimicrobial resistance (AMR) is defined as the continuation of the growth of a microorganism in presence of an antimicrobial substance at a concentration that is expected to inhibit its growth. In other words, the bacterium that was unable to grow in presence of an antibiotic at a certain concentration can do so by developing novel characteristics. Antibiotic resistance can be an intrinsic feature of some groups of bacteria that can be attributed to the presence of certain genes enabling them to survive in presence of the antibiotic (Balaban et al. 2019; Blair et al. 2015; Schrader et al. 2020). However, a large number of bacteria can acquire the antibiotic-resistant phenotype by incorporating certain mutations or by capturing already existing antibiotic resistance genes via horizontal gene transfer (HGT) mechanisms (Allen et al. 2010; Blair et al. 2015). While rampant HGT events can help in acquiring antibiotic resistance in a singlestep evolutionary process, the acquisition of mutations in the genome may take several generations to develop at a population level. Nevertheless, considering the natural rate of mutations at one per million bases, the rate of growth of the bacterial culture or a short generation time, and the vast number of cells in a culture vessel, resistance to any antibiotic develops in a very short time.

In the conventional antibiotic drug development process, the drugs are targeted against the vital metabolic processes in the bacterial cell, therefore, creating an evolutionary selection pressure that hastens the emergence of the resistant phenotype. The most important pathways targeted are the synthesis of the 
bacterial cell wall, protein synthesis, DNA replication, and transcription processes. Consequently, the resistance mechanisms developed by the bacterial cells are specifically targeted to these pathways. Broadly, the antibiotic resistance mechanisms in the bacterial cell can be categorized into the following four groups: (a) modification of the antibiotic target site, (b) enzymatic inactivation of the antibiotic, (c) prevention of antibiotic access to the target, and (d) modification of the metabolic pathways (Blair et al. 2015). Though the details of the mechanisms are understood to a great extent, the discussion about the mechanisms is beyond the scope of this article.

Genetic mechanisms are largely attributed for the development of antibiotic resistance, however, recent developments in the area of single-cell biology, population-level studies involving microfluidic devices, and microscopy, has increased our understanding of a primarily phenotypic concept called persistence among the bacteria where the bacteria even though not genetically resistant to an antibiotic, can still survive in its presence by modulating its growth pattern. Persistent bacteria are a sub-population of the cells that have stopped growing in presence of the antibiotic. Once the antibiotic treatment is withdrawn, they can regrow normally. With the persistent population, the MIC of a particular antibiotic remains unchanged, therefore it is not a heritable phenomenon and hence not genetic, however, it has a lot of clinical significance that contributes to the persistent infections and clinical failure of antibiotic treatments (Balaban 2004; Balaban et al. 2019; Harms et al. 2016; Schrader et al. 2020).

As mentioned earlier, traditional antibiotic targets are essential pathways in the bacterial cell leading to the selection of resistant phenotypes. However, recently there has been increasing interest to find drugs that are "evolution proof" to which resistance would not develop quickly (Bell and MacLean 2018). There is also a growing interest to develop drugs that are anti-virulence in nature, meaning, they would not target any essential pathways in the bacterial cell, rather the virulence factors would be targeted to reduce the overall disease outcome (Carabajal et al. 2019; Curtis et al. 2014; Defoirdt 2018; El-Halfawy et al. 2020; Ogawara 2021). Also, novel drug combination therapies are being tested against pathogens to design the best treatment regimens (Barbosa et al. 2018; Brennan-Krohn et al. 2018; Lin et al. 2019; Olsson et al. 2020). In this context, the role of polymyxin drugs is very interesting considering their mode of action and the development of resistance.

\section{Polymyxins: The last-resort antibiotics}

The emergence of novel MDR bacterial pathogens has necessitated looking into developing new treatment strategies including the use of old drugs that have been discontinued because of toxicity issues (Biswas et al. 2012; Trimble et al. 2016). The polymyxin group of antibiotics discovered in 1947 from the bacterium Bacillus polymyxa are one such candidate. Till now, more than fifteen varieties of polymyxins have been isolated and characterized, among which the most prominent ones are polymyxin $\mathrm{B}$ and polymyxin $\mathrm{E}$ (also known as colistin) (Mmatli et al. 2020; Nang et al. 2021; Poirel et al. 2017a; Trimble et al. 2016). The polymyxins exhibit significant inhibitory effect against the Gram-negative bacterial pathogens, therefore, it has emerged as an ultimate choice to target such pathogens that have already become resistant to the frontline antibiotics such as $\beta$-lactams, aminoglycosides, and the fluoroquinolones (Biswas et al. 2012; Landman et al. 2008; Mmatli et al. 2020; Nang et al. 2021; Poirel et al. 2017a). Hence polymyxins are considered to be the last-resort antibiotic to be used against MDR Gram-negative pathogens.

At the structural level, polymyxins are very similar to the cationic antimicrobial peptides (CAMPs) produced by the eukaryotes as a first line of defence against pathogens. These are lipopeptides in nature with an approximate mass of $1.2 \mathrm{kDa}$. Polymyxins have a typical ring and tail structure (figure 1A), where the ring is made up of a polycationic peptide and the tail made up of tripeptide chain attched with fatty acids (Nation et al. 2014; Newton 1956; Poirel et al. 2017a; Trimble et al. 2016; Yu et al. 2015). The polymyxin B and colistin differ at a single amino acid residue in the peptide ring where D-phenylalanine is replaced by a D-leucine residue in colistin (Landman et al. 2008; Yu et al. 2015). While the cationic peptide ring provides a hydrophilic nature to the polymyxins, the fatty acyl chain is hydrophobic. This structural feature is generally attributed to the ability of the polymyxins to insert into the cell membranes leading to its disintegration (Nang et al. 2021; Nation et al. 2014; Trimble et al. 2016). This typical feature is also responsible for the observed nephrotoxicity in humans. The polymyxins are synthesized by the non-ribosomal peptide synthetases and are therefore referred to as the non-ribosomal peptides.

Polymyxin B and colistin have similar antibacterial properties against the Gram-negative bacterial species and are specifically used against infections caused by the MDR pathogens such as $K$. pneumoniae, $P$. 

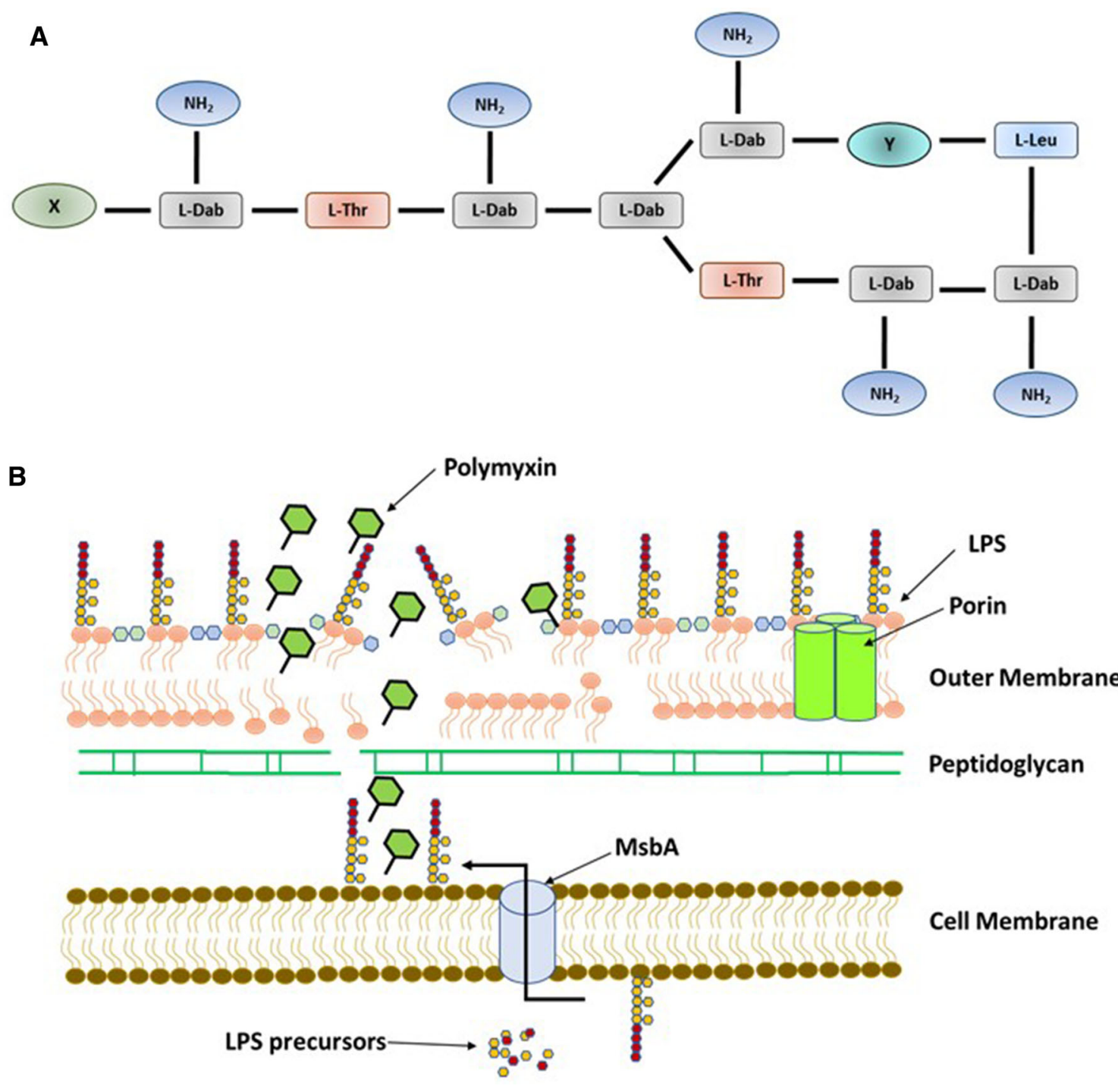

Figure 1. The structure of polymyxins and the possible mode of action. (A) Structure of the polymyxin B molecule. In colistin, a D-leucine residue replaces the D-Phe residue (denoted as Y) in the cyclic peptide region of the polymyxin backbone. X- fatty acid chain, Dab- diamino-butyric acid. (B) The model of the polymyxin- bacterial membrane interaction. Polymyxin molecules (shown as a hexagon with a tail) when interacting electrostatically with the bacterial outer membrane lipopolysaccharide the $\mathrm{Mg}^{2+}$ and $\mathrm{Ca}^{2+}$ ions (small hexagons attached to the LPS) that stabilize the membrane structure are displaced leading to the insertion of the polymyxin molecule into the membrane. This process destabilizes the membrane integrity. After this event, the polymyxin can also interfere in the assembly of the nascent LPS molecules at the cell membrane and their transport to the outer membrane. The overall process helps in the disintegration of the bacterial membrane leading to cell lysis and death (see the text for details).

aeruginosa, and A. baumannii as the last option (Nang et al. 2021; Nation et al. 2014; Trimble et al. 2016). As a primarily membrane-targeting antibiotic, polymyxins show limited activity against Gram-positive pathogens considering the presence of a thick peptidoglycan layer and the absence of the outer membrane (OM) (Yin et al. 2020). Polymyxins cause cell damage leading to cell death by disrupting the membrane integrity. This mechanism is also at play when the polymyxins interact with the membranes of the human cells, disintegrating them leading to toxicity. Primarily, toxicity is observed in the kidney and brain tissues, though more severity is observed in the renal tissues, thereby, leading to the prolonged discontinuity in their use till the mid-1990s (Nang et al. 2021). Recent chemical modifications in the drug moiety have been attempted to reduce toxicity. Also, changes in the dosage, modifications in the formulations have reduced the toxicity level to a great extent. Polymyxin B is shown to be less toxic than its prodrug colistimethate, therefore can be 
administered directly. However, colistin is generally administered as prodrug colistin methanesulfonate that undergoes hydrolysis to produce the colistin (Nang et al. 2021; Nation et al. 2014; Poirel et al. 2017a; Trimble et al. 2016).

\subsection{Mode of action}

Considering the presence of the cationic peptide ring and the hydrophobic fatty acyl chain, the polymyxins act by disrupting the membrane integrity leading to the permeabilization of the bacterial cell. Though this mode of action is plausible because of the typical structure of the drug, however, there are several hypotheses/models about multiple modes of activity of the drug responsible for its bactericidal activity (Nang et al. 2021; Poirel et al. 2017a; Trimble et al. 2016; Yu et al. 2015). The most important pathways of polymyxin activity are as follows.

3.1.1 Membrane lysis pathway: Since the discovery and the initial uses of polymyxins, the membrane lysis pathway has been proposed as the most important mechanism by which polymyxins can exert their effect. The ability of the polymyxins to be associated with the membranes and thereby disrupting the osmotic balance of the cell is considered to be the primary mechanism (Newton 1956). The selective targeting of the Gramnegative bacteria by polymyxins can be attributed to the presence of the outer membrane (OM). Also, investigations have shown that Gram-negative bacterial cells pre-treated with polymyxins became more susceptible to the treatment of lysozyme and the peptidoglycan targeting $\beta$-lactam antibiotics, indicating the role of the polymyxin-membrane interaction preceding peptidoglycan lysis (Brennan-Krohn et al. 2018; MacNair et al. 2018).

In the Gram-negative bacteria, as per the current understanding of the polymyxin cell membrane interaction, the cationic peptide region binds electrostatically to the negatively charged lipopolysaccharide (LPS) molecules of the bacterial OM. At the same time, the hydrophobic fatty acid chain of polymyxin interacts with the lipid A of the LPS. These interactions lead to the displacement of the membrane-stabilizing cations such as $\mathrm{Ca}^{2+}$ and $\mathrm{Mg}^{2+}$ and the "self-promoted uptake" of the polymyxin into the OM (Ayoub Moubareck 2020; Evans et al. 1999; Fernández et al. 2013; Landman et al. 2008; Trimble et al. 2016). At this point, the OM becomes weakened and the permeability barrier is breached leading to the release of the periplasmic proteins and the breakage of the membrane (figure 1B) (Hancock 1984; Trimble et al. 2016; Yu et al. 2015). In the subsequent step, polymyxin interacts with the cell membrane (CM), though there is no consensus about the mechanism of action at the $\mathrm{CM}$ level (Landman et al. 2008; Yu et al. 2015). Previous studies have indicated that polymyxin B treatment results in lipid exchange between the outer and inner membranes of the Gram-negative bacteria, leading to the loss of specificity in the phospholipid composition (Berglund et al. 2015; Clausell et al. 2007; Yu et al. 2015). The inner leaflet of the OM and the CM, largely made up of the anionic phospholipids, when interacting with the cationic polymyxin, undergo disruption causing osmotic imbalance leading to cell lysis ( $\mathrm{Yu}$ et al. 2015).

Even though several studies indicate that polymyxin interaction with the bacterial membranes leading to their lysis is the primary mechanism by which polymyxin acts, reasonable gaps exist in our understanding if mere interaction with the membrane is sufficient to cause cell death (Trimble et al. 2016; Velkov et al. 2010). A recent study (Sabnis et al. 2021) indicated that colistin can easily permeabilize the bacterial CM that can lead to cell death. Using E. coli expressing the plasmid-encoded $\mathrm{mcr}$ - 1 , this study showed that colistin can easily penetrate the $\mathrm{OM}$, however, the $\mathrm{CM}$ can resist the onslaught of colistin. In contrast, E. coli carrying the empty plasmid can easily be breached leading to cell death (Sabnis et al. 2021). This work also unequivocally demonstrated that the LPS of both $\mathrm{OM}$ and $\mathrm{CM}$ are the targets of colistin. However, the LPS modified with phosphoethanolamine (PEtN) in the $\mathrm{CM}$ is more protective towards colistin treatment. This also proves the previous finding that in comparison to the OM, the CM contains a hundred times fewer LPS (Osborn et al. 1972; Sabnis et al. 2021), therefore, significantly a smaller number of colistin targets at the CM. These authors using murepavadin, a drug that inhibits the transport of LPS from the CM to OM, have shown that the $P$. aeruginosa can be re-sensitized to colistin treatment (Sabnis et al. 2021), suggesting that the accumulation of nascent LPS at the CM makes it more susceptible to colistin action.

Notwithstanding the above, it has been shown previously in $P$. aeruginosa and $E$. coli treated with polymyxin at concertation greater than the MIC, the proportion of cell death increases drastically, whereas the amount of cell membrane disruption increases marginally (Daugelavicius et al. 2000; Zhang et al. 2000). These data suggest the existence of multiple 
alternative mechanisms of polymyxin activity responsible for the death of the bacterial cell.

Some of the alternative mechanisms suggested for the polymyxin mode of action are as follows.

\subsubsection{Inhibition of bacterial respiration: Polymyxin is} shown to inhibit bacterial respiration in some of the models studied. Disruption of the cell membrane caused by the polymyxins can affect cellular respiration adversely, as an intact cell membrane is a prerequisite for this physiological process in the bacteria (Ayoub Moubareck 2020; Nang et al. 2021; Storm et al. 1977; Trimble et al. 2016). Interestingly, polymyxins have also been shown to affect the normal respiratory process in the Gram-positive bacterium Bacillus subtilis during the sporulation process. Specifically, polymyxin inhibits the NADH oxidase and the NADH cytochrome c reductase enzymes (Tochikubo et al. 1986). In Mycobacterium smegmatis too, polymyxin inhibits NADH dehydrogenase ultimately affecting respiration (Mogi et al. 2009).

Bacterium Streptococcus pyogenes is known to be susceptible to polymyxin unlike many other Grampositive bacterial species (Olaitan et al. 2014; Trimble et al. 2016). Polymyxin exerts its inhibitory effect in $S$. pyogenes by targeting the fatty acid biosynthetic pathway leading to the activation of the stringent response. Interestingly, polymyxin treated $S$. pyogenes cells show an increase in the concentration of the guanosine tetraphosphate (ppGpp) alarmone, whose accumulation in the cell can be attributed to the inactivation of the SpoT enzyme, because of the decrease in the cellular ATP level (Cortay and Cozzone 1983a,, b; Storm et al. 1977; Trimble et al. 2016). This mechanism of inhibition of cellular respiration is consistent with the effect exerted by other cationic antimicrobial peptides (Hancock and Chapple 1999; Spindler et al. 2011). Though inhibition of cellular respiration is not primarily responsible for cell death, polymyxin contributes to it by affecting cellular energetics.

\subsubsection{Generation of reactive oxygen species (ROS):}

Polymyxin treatment can also lead to the generation of reactive oxygen species (ROS) as suggested by several recent studies. ROS such as superoxide $\left(\mathrm{O}_{2}{ }^{-}\right)$, hydrogen peroxide $\left(\mathrm{H}_{2} \mathrm{O}_{2}\right)$, and hydroxyl radical $\left({ }^{\circ} \mathrm{OH}\right)$ can lead to the oxidative damage of the cellular components such as lipids, DNA, and proteins resulting in cell death (Ayoub Moubareck 2020; Imlay 2015; Kohanski et al. 2007; Yu et al. 2015). However, this mechanism is not fully accepted yet considering experimental observation of ROS independent killing of cells by colistin (Brochmann et al. 2014). All these conflicting results and hypotheses need reconciliation by more comprehensive studies to find out the role of ROS in the overall cell growth inhibition and death.

3.1.4 Ribosome binding: Polymyxins have been shown to precipitate the ribosomes in the $E$. coli cells (Nakajima and Kawamata 1966; Teuber 1967) indicating their interaction. This is similar to what has been observed using cationic aminoglycoside antibiotics that bind to the ribosomes (McCoy et al. 2013). Fluorescence resonance energy transfer (FRET) assay demonstrated that polymyxin indeed interacts with the 16S rRNA in the A-site of the E. coli ribosome, albeit with a lower affinity than the kanamycin for the above site (Hancock 1997). However, the significance of this interaction is not fully elucidated, and no conclusive evidence is available to suggest any influence on protein synthesis in the bacterial system. Interestingly, eukaryotic translation is known to be inhibited by polymyxin as observed in vitro (McCoy et al. 2013; Trimble et al. 2016).

3.1.5 Effect on cell division: Cell division is not the primary cellular function that is affected by the polymyxins, however, experimental evidence suggests that treatment with a sublethal concentration of colistin in $P$. aeruginosa reduces the number of dividing cells significantly resulting in a reduction in the number of colony-forming units (Mortensen et al. 2009). Though this does not result in the significant killing of the cells, it resulted in increased rigidity of the cell. Therefore, it may be argued that colistin binds to the peptidoglycan affecting the cell division. The bacterial cell division is a very dynamic process involving a plethora of different factors functioning at a spatial and temporal dimension. Alternatively, polymyxin may bind with some of these factors resulting in the arrest of cell division. It has also been proposed that polymyxin and cell membrane interaction can negatively impact the segregation of the duplicated chromosome before the cytokinesis (David and Rastogi 1985). Though these results suggest some leads, our understanding of polymyxin affecting the cell division remains patchy at the moment and the details are inconclusive.

3.1.6 Gram-positive secretion system: Gram-positive bacterial species though are largely unaffected by the polymyxin, $S$. pyogenes is one species that shows polymyxin sensitivity, which can be attributed to the absence of the gene $m p r F$ responsible for modifying the negative charge of phosphatidylglycerol on the 
capsular polysaccharide (Vega and Caparon 2012). It has also been observed that the $S$. pyogenes ExPortal system, a part of the Sec secretion system located close to the site of cell division, is targeted by the polymyxins (Vega and Caparon 2012). The sublethal concentration of polymyxin inhibited the secretion of cysteine protease $\mathrm{SpeB}$, and the streptolysin $\mathrm{O}$, by disrupting the lipid structure around the ExPortal (Rosch et al. 2007; Vega and Caparon 2012). Interestingly, this experiment did not detect any membrane damage leading to the conclusion that this could be one of the several types of secondary effects the polymyxins have on the cell.

In summary, polymyxins primarily target the bacterial membrane. However, this is not sufficient to explain the observed lethality exerted by them. Several secondary mechanisms as discussed above certainly play a role in the overall inhibitory action of the polymyxins.

\subsection{Polymyxin resistance}

As the clinical use of polymyxin was stopped due to the toxicity issues there are not many studies conducted initially to determine the emergence of polymyxin resistance. However, in recent times there is renewed interest to understand the mechanisms of resistance to polymyxins as this remains our last hope antibiotic. The primary way by which bacteria become resistant to polymyxins is by modifying the membrane structure to reduce the interaction with the polymyxins. This is primarily accomplished by modifying the charge on the LPS moiety of the membrane to repel the cationic polymyxins. Other mechanisms can also contribute to the development of resistance such as drug efflux, decreased uptake, and production of capsules, etc.

Polymyxin resistance among the bacterial species can be looked at from two perspectives- intrinsic resistance and acquired resistance that are discussed in the following sections.

3.2.1 Intrinsic resistance to polymyxins: There are a few Gram-negative bacterial species known to be naturally resistant to polymyxins such as Proteus mirabilis, Moraxella catarrhalais, Helicobacter pylori, Serratia marcescens, Providencia spp., Burkholderia cepacia, Edwardsiella tarda, etc. (Aquilini et al. 2014; Biswas et al. 2012; Jiang et al. 2010; Landman et al. 2008; Lin et al. 2014). Primarily the addition of the cationic groups such as phosphoethanolamine (PEtN) and the 4-amino-4-deoxy-L-arabinose (L-Ara4N) to the
LPS moiety of the outer membrane contributes towards the resistance to polymyxins in these organisms (Raetz et al. 2007; Simpson and Trent 2019). This results in reducing the overall negative charges of the membrane that can no more bind to the polymyxin leading to resistance (Olaitan et al. 2014; Poirel et al. 2017a; Trimble et al. 2016). In these organisms, the operon $\operatorname{arnBCADTEF}$ and the gene eptB are constitutively expressed leading to the addition of PEtN and L-Ara4N to the LPS (Poirel et al. 2017a). This pathway is discussed in the following sections.

3.2.2 Acquired resistance to polymyxins: The acquired resistance towards the polymyxins generally involves the modification of the LPS to reduce the polymyxinouter membrane interaction. Resistance to polymyxins can emerge by the modulation of genes involved in the LPS modification pathways. These pathways are generally regulated by the bacterial sensory modules such as two-component signal transduction systems (TCSs). Therefore, after sensing polymyxins the TCSs systems can direct the modification of the LPS leading to resistance. Resistance can also emerge by the acquisition of plasmids (mobile colistin resistance or $\mathrm{mcr}$ ) encoding genes for polymyxin resistance, by horizontal gene transfer events. These mechanisms are addressed in the following sections.

\subsubsection{Two-component signal transduction system}

(TCS) mediated resistance to polymyxins: Bacterial TCSs were first reported almost three decades ago highlighting their role in sensing the environmental cues and responding to the changes by modulating the expression of several genes (Stock et al. 2000). Almost all bacterial species possess several of these signal transduction systems in their genome; their numbers are related to the genome size and the range of environmental niches the organism lives (Bem et al. 2015; Bhagirath et al. 2019; Tierney and Rather 2019). Bacterial TCSs consisting of a sensor histidine kinase (HK) get autophosphorylated after sensing an external signal, which relays the signal to the response regulator (RR) by phosphorylating it at a conserved aspartic acid site (Stock et al. 2000). The activated response regulator acts as a transcription factor and affects the transcription of several genes. There are several TCSs involved in the polymyxin resistance pathway specifically in modifying the LPS (Table 1) (Huang et al. 2020; Simpson and Trent 2019). In our discussion, two TCSs, PhoPQ and PmrAB, which are studied in great detail will be addressed. PhoPQ and PmrAB TCSs are 
Table 1.. Two-component signal transduction systems (TCSs) that are involved in the polymyxin resistance pathways in different Gram-negative bacterial pathogens

\begin{tabular}{|c|c|c|c|}
\hline $\begin{array}{l}\text { Two-component } \\
\text { systems (TCSs) }\end{array}$ & Pathways regulated & Bacterial species & References \\
\hline \multirow[t]{4}{*}{ PhoPQ } & \multirow{4}{*}{$\begin{array}{l}\text { Lipid A modification via the addition } \\
\text { of L-Ara4N and PEtN }\end{array}$} & E. coli & Moon and Gottesman 2009 \\
\hline & & S. Typhimurium & Gunn et al. $1998 \mathrm{a}$ \\
\hline & & P. aeruginosa & Macfarlane et al. 2000 \\
\hline & & A. hydrophila ${ }^{*}$ & Liu et al. 2021 \\
\hline \multirow[t]{5}{*}{ PmrAB } & \multirow{5}{*}{$\begin{array}{l}\text { Lipid A modification via addition } \\
\text { of L-Ara4N and PEtN }\end{array}$} & P. aeruginosa & McPhee et al. 2003 \\
\hline & & $S$. Typhimurium & Gunn et al. $1998 b$ \\
\hline & & A. baumannii & Arroyo et al. 2011 \\
\hline & & K. pneumoniae & Choi and Ko 2014 \\
\hline & & E. coli & Trent et al. 2001 \\
\hline ParRS & $\begin{array}{l}\text { Lipid A modification via addition } \\
\text { of L-Ara } 4 \mathrm{~N} \text { and PEtN }\end{array}$ & P. aeruginosa & Fernández et al. 2010 \\
\hline CprRS & $\begin{array}{l}\text { Lipid A modification via addition } \\
\text { of L-Ara4N and PEtN }\end{array}$ & P. aeruginosa & Fernández et al. 2012 \\
\hline ColRS & $\begin{array}{l}\text { Lipid A modification via addition } \\
\text { of L-Ara4N and PEtN }\end{array}$ & P. aeruginosa & Gutu et al. 2013 \\
\hline CbrAB & $\begin{array}{l}\text { Lipid A modification via addition } \\
\text { of L-Ara4N and PEtN }\end{array}$ & P. aeruginosa & Yeung et al. 2011 \\
\hline CrrAB & Modulation of PmrAB & K. pneumoniae & Wright et al. 2015 \\
\hline Rcs system & $\begin{array}{l}\text { Lipid A modification via addition } \\
\text { of L-Ara } 4 \mathrm{~N} \text { and PEtN }\end{array}$ & S. Typhimurium & $\begin{array}{l}\text { Mouslim and } \\
\text { Groisman } 2003\end{array}$ \\
\hline VprAB & Glycosylation of lipid A & V. cholerae & Herrera et al. 2014 \\
\hline CarRS & Glycosylation of lipid A & V. cholerae & Bilecen et al. 2015 \\
\hline EnvZ/OmpR & $\begin{array}{l}\text { Lipid A modification via addition } \\
\text { of L-Ara } 4 \mathrm{~N} \text { and modulation of PhoPQ }\end{array}$ & A. hydrophila & Liu et al. 2021 \\
\hline
\end{tabular}

\footnotetext{
${ }^{*}$ In A. hydrophila PhoPQ is not primarily involved in the colistin resistance pathway. However, in the absence of EnvZ/ OmpR, PhoPQ system is activated.
}

present in most Gram-negative pathogens. Though at the molecular level there are few functional differences, for simplicity we will present a general picture for a better understanding of their role.

PmrAB and PhoPQ mediated LPS modification has been studied thoroughly in the Gram-negative bacterial pathogen Salmonella enterica serovar Typhimurium (S. Typhimurium) (Chen and Groisman 2013; Groisman et al. 2021; Huang et al. 2020). Therefore, this bacterium serves as a model to understand the mechanism of resistance to cationic antimicrobial peptides (CAMPs) including polymyxins. The overall mechanism of signal transduction in the PhoPQ and PmrAB and the gene regulation leading to polymyxin resistance is depicted in figure 2. Briefly, the PhoPQ consisting of the membrane located PhoQ sensor kinase senses the environmental signals such as low magnesium $\left(\mathrm{Mg}^{2+}\right)$, low $\mathrm{pH}$ ( $\mathrm{pH}$ 5.5), CAMPs, and polymyxins, etc., and gets autophosphorylated that in turn activates the PhoP response regulator. The activated PhoP upregulates the expression of the gene
pagL that deacylates lipid A leading to increased hydrophobicity of the LPS resulting in less penetration of polymyxins (Han et al. 2018). Phosphorylated PhoP inhibits the expression of eptB via a small RNA regulator $m g r R$ that is responsible for adding the PEtN to the Kdo (3-deoxy-D-manno-octulosonic acid) residue in the LPS (Olaitan et al. 2014; Poirel et al. 2017a). The significance of this modification is not completely understood from the polymyxin resistance point of view. The evidence though exists for the presence of other sRNAs such as MicA that may have a role in the overall regulation of the PhoPQ system (Olaitan et al. 2014). The phosphorylated PhoP also activates the second TCS PmrAB via a connector $p m r D$. The PmrD via protein-protein interaction stabilizes the phosphorylated PmrA by inhibiting its dephosphorylation. The PmrA response regulator activates several operons involved in the LPS modification pathways such as arnBCADTEF (L-Ara4N addition to LPS), pmrCAB (PEtN addition to lipid A), cptA (PEtN addition to LPS core), $p m r R$ (phosphorylation of lipid A) (figure 2) 


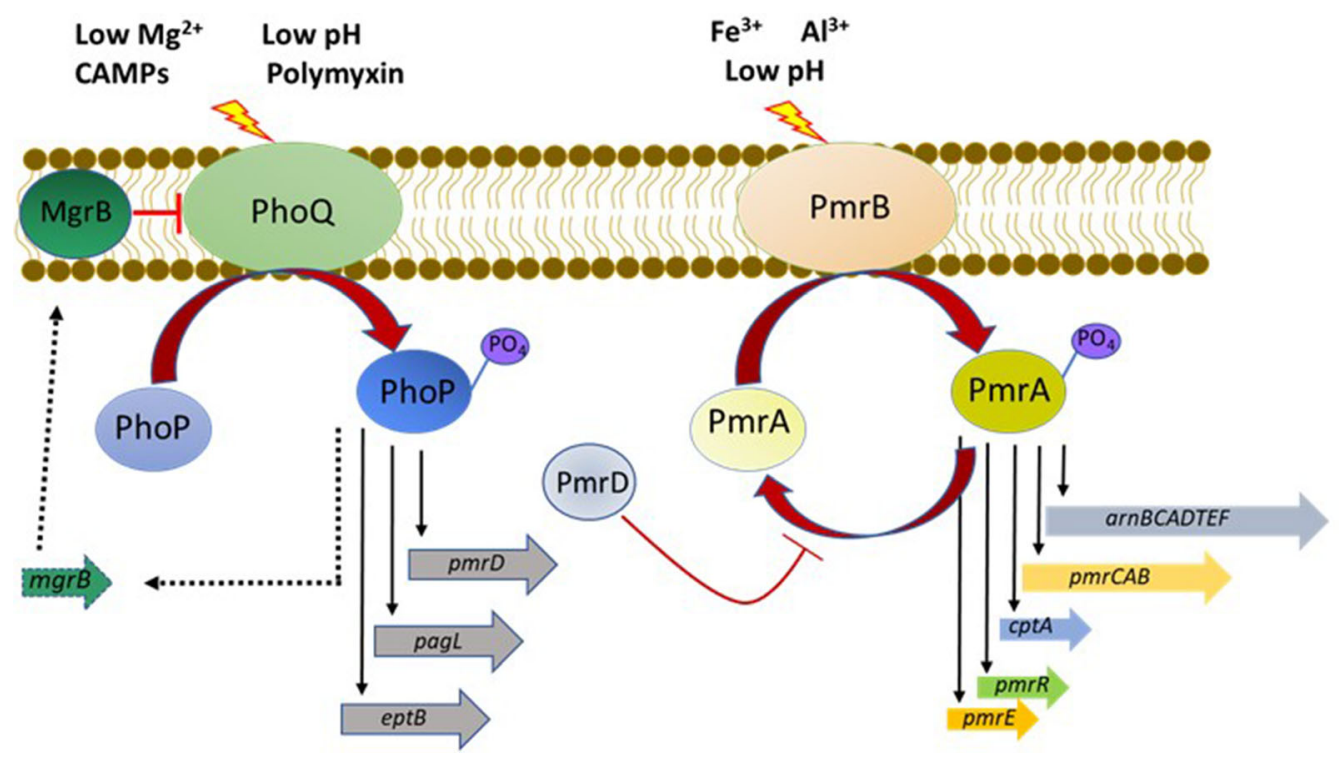

Figure 2. Bacterial two-component signal transduction system (TCS) mediated lipopolysaccharide (LPS) modification pathway leading to polymyxin resistance. The PhoPQ gets activated after sensing the environmental cues (low $\mathrm{Mg}^{2+}, \mathrm{Ca}^{2+}$, CAMPS, and polymyxin, etc.) and in turn, activates the PmrA response regulator of the PmrAB TCS via PmrD protein. The PmrA activates several genes and operons that modify the LPS by adding positively charged PEtN and L-Ara4N. This modification ultimately restricts the interaction of the membrane with the polymyxin thereby avoiding its toxic effects. The $\mathrm{PmrB}$ sensor kinase also gets activated sensing cations such as $\mathrm{Fe}^{3+}$ and $\mathrm{Al}^{3+}$ as well as low $\mathrm{pH}$ conditions and can activate the cognate response regulator PmrA. This is a general mechanism of LPS modification via bacterial TCS that is reported in E. coli and $S$. Typhimurium. Variations to this general mechanism are observed in the case of other Gram-negative pathogens. As shown here in the case of $K$. pneumoniae, the PhoP also modulates the expression (shown as dashed arrows) of a membrane located small protein MgrB that can inhibit the activity of PhoQ sensor kinase. This negative feedback mechanism helps in the reset process of the whole pathway (see the text for details).

(Table 1) (Nang et al. 2021; Olaitan et al. 2014). The activation of all these operons and genes converges in the LPS making it more and more positively charged resulting in the polymyxin resistance phenotype.

Though the TCS-mediated LPS modification pathway looks quite straightforward, several variations in different organisms can be found that can be attributed to their presence in different host environmental niches, pathogenesis mechanisms, and the host innate immune response. Interestingly, polymyxin-resistant bacterial pathogens exhibit mutations in the genes encoding PhoPQ, PmrAB, and the operons they regulate, leading to their constitutive expression and LPS modification. There are hundreds of mutations documented in different organisms responsible for LPS modification leading to polymyxin resistance (Huang et al. 2020; Liu et al. 2021; Olaitan et al. 2014; Poirel et al. 2017a).

Apart from these two TCSs, many bacteria possess multiple TCSs that possibly crosstalk and ultimately coordinate in the LPS modification. For example, in the $P$. aeruginosa, six TCS modules are involved in the polymyxin resistance pathways such as PhoPQ, PmrAB, ColRS, CprRS, ParRS, and CbrAB (Fernández et al. 2010, 2012; Gutu et al. 2013; Macfarlane et al. 2000; McPhee et al. 2003; Yeung et al. 2011). There is quite a possibility that these TCSs sense different environmental cues and get activated in different circumstances, however, they all respond similarly by activating the LPS modifying genes (Table 1) (Fernandez and Hancock 2012; Gutu et al. 2013; Olaitan et al. 2014). Similarly, in the case of $K$. pneumoniae an additional TCS CrrAB has been reported to be involved in the polymyxin resistance pathway (McConville et al. 2020; Wright et al. 2015) along with the PmrAB and PhoPQ systems. Interestingly, the CrrAB TCS operates via modulating the PmrAB system (Cheng et al. 2016). K. pneumoniae also possesses a negative regulator $(\mathrm{MgrB})$ of $\mathrm{PhoQ}$ kinase, inactivation of which can lead to upregulation of the PhoPQ system (figure 2) (Cannatelli et al. 2014; Poirel et al. 2015). It can be argued that these complex regulatory architecture among the bacteria has evolved to integrate 
a diverse range of environmental stimuli to a common response by the modification of the outer membrane.

In the pathogen $A$. baumannii, a very interesting phenomenon is observed concerning the polymyxin resistance, where the complete LPS is lost from the bacterial cell making it resistant to polymyxins. This happens because of the insertional inactivation by the ISAba11 element in the lipid A biosynthetic cluster involving the genes $\operatorname{lp} x A, \operatorname{lp} x C$, and $\operatorname{lp} x D$ (Moffatt et al. 2011, 2019, 2010). As the lipid A synthesis is stopped, the polymyxin target is no more available in the $A$. baumannii cells leading to a very high level of resistance. Interestingly, in A. baumannii PmrAB is the only TCS that is involved in the polymyxin resistance pathway (Nang et al. 2021).

In the aquatic bacterial pathogen Aeromonas hydrophila, resistance to polymyxins is not so prevalent. However, with the indiscriminate use of polymyxins in aquaculture, resistance is increasingly being reported (Gonzalez-Avila et al. 2021). In A. hydrophila, the primary way by which colistin resistance develops is by increased expression of the TCS EnvZ/OmpR that regulates the LPS modification pathway via the addition of L-Ara4N moiety to the lipid A (Liu et al. 2021). It has also been observed that EnvZ/OmpR regulates the expression of an outer membrane protein (encoded by the gene 3832) which is predicted to be an autotransporter possibly involved in colistin uptake. Expression of this autotransporter is upregulated in presence of colistin, whereas deletion of the EnvZ/ OmpR TCS abolishes its expression. Another significant difference concerning A. hydrophila colistin resistance is that the TCS PhoPQ does not play a primary role in colistin resistance. However, in the absence of the EnvZ/OmpR, PhoPQ gets activated and modify the LPS via PEtN modification (Liu et al. 2021). It remains to be discovered how EnvZ/OmpR system is connected to PhoPQ at the molecular level, though the existence of a protein connector between the two TCSs cannot be ruled out.

Apart from the TCSs some bacteria also employ various other strategies to mount resistance to polymyxins. Important among them is the formation of biofilms at the site of infections, in the medical devices, and their environmental niche. Biofilms are considered to be a response to the stress and the biofilm architecture is such that it makes the biofilm residents recalcitrant to very high concentrations of antibiotics including polymyxins. In $P$. aeruginosa, psrA, and cbrA, and in Vibrio cholerae the carR genes are found to be regulating the biofilm formation as well as developing polymyxin resistance (Bilecen et al. 2015;
Gooderham et al. 2008). K. pneumoniae is known to produce a lot of capsular polysaccharides that can use them to trap antimicrobial peptides including polymyxins reducing their access to the outer membrane leading to resistance development (Llobet et al. 2008), though this mechanism is still not fully understood.

Bacterial efflux pumps are very important to expel the accumulated noxious substances inside the cell. Many of these efflux pumps also contribute to the polymyxin resistance pathway. The efflux pumps such as AcrAB-TolC, MtrC-MtrD-MtrE, RosAB, KpnEF, and $\mathrm{Vex} A B$, etc. are shown to be responsible for developing tolerance to polymyxins in several bacterial pathogens (Bengoechea and Skurnik 2000; Bina et al. 2008; Fehlner-Gardiner and Valvano 2002; Padilla et al. 2010; Srinivasan et al. 2014; Tsai et al. 2012; Tzeng et al. 2005; Warner and Levy 2010). In polymyxin B resistant $E$. coli strains the marRAB operon has been shown to upregulate the AcrAB-TolC efflux pump (Warner and Levy 2010). Apart from the efflux systems, the outer membrane porins (OMPs) are also shown to be involved in polymyxin resistance most likely by limiting the uptake (Mathur and Waldor 2004).

3.2.4 Plasmid-mediated polymyxin resistance: For quite a long time, it was accepted that bacteria become resistant to polymyxins via different adaptation mechanisms including the LPS modification pathways. However, a plasmid-mediated gene $\mathrm{mcr}-1$ (mobilized colistin resistance gene) was detected in the E. coli and $K$. pneumoniae isolates from China during the years 2011-2014 (Liu et al. 2016). The $m c r-1$ gene encodes a protein that is homologous to the phosphoethanolamine (PEtN) transferase enzyme involved in the LPS modification pathway. This finding of the plasmid-borne gene $m c r-1$ is quite significant considering that it could be acquired horizontally leading to the rapid development of polymyxin resistance (Ayoub Moubareck 2020; Mmatli et al. 2020; Poirel et al. 2017b). As $\mathrm{mcr}$ 1 expression alone increases the MIC of polymyxin by 4- to 8-fold, a single step of acquisition of the plasmid can make the susceptible bacteria resistant.

Since the initial discovery of the $m c r-1$ in China, this gene has been reported from across the world. There is strong evidence that the emergence of the plasmid encoding $m c r-1$ gene correlates with the widespread use of polymyxins in farm animals as a growth supplement several years ago. Therefore, it is quite possible for the rapid transmission of the plasmid along with the food supply. This gene is associated with the 
Enterobacteriaceae isolated from the environment, food, meat products, animals, and humans (Poirel et al. 2017a). Since the first report of $\mathrm{mcr}$ - 1 nine more $\mathrm{mcr}$ genes ( $m c r-2$ to $m c r-9)$ encoded on different plasmids have been reported (Ayoub Moubareck 2020; Carroll et al. 2019; Gonzalez-Avila et al. 2021; Mmatli et al. 2020). The various $m c r$ genes act similarly and are classified based on their sequence divergence from each other. The $m c r-1$ is the most prevalent type in the environment. Considering its widespread occurrence around the world, it could make polymyxins ineffective if urgent steps are not taken to curb the use of polymyxins in animal farming.

\subsection{Potential solutions to polymyxin resistance}

The emergence of resistance to different drugs including polymyxin is inevitable considering it is a normal physiological and evolutionary process. Therefore, to keep our last resort antibiotic useful for times to come, its use must be rationalized to prolong the development and spread of resistance. Meanwhile, there have been several novel methods that can be considered to develop new drugs, as well as the use of combinatorial treatment approaches must be adopted. Some of these approaches are described as follows.

3.3.1 Bacterial TCS as novel drug targets: Studies in several bacterial species have identified dedicated TCS modules that are implicated in the regulation of genes involved in antibiotic resistance pathways. In the case of $S$. aureus, TCSs such as BraSR, VraSR, GraSR, VanSR are responsible for developing resistance against bacitracin, vancomycin, and several other CAMPs (Bem et al. 2015; Depardieu et al. 2007; Fridman et al. 2013; Hiron et al. 2011). In M. tuberculosis the TCS MtrAB is implicated in the multidrug resistance phenotype (Nguyen et al. 2010). Similarly, in the cases of Enterobacteriaceae, TCSs such as BaeSR, ArcBA, PhoPQ, PmrAB, PhoBR, CpxAR, LysR, etc. have been found to contribute to the drug resistance against various classes of antibiotics such as $\beta$-lactams, aminoglycosides, fluoroquinolones, tetracyclines, polymyxins, and several CAMPs (Bem et al. 2015; Doddangoudar et al. 2011; Fernandez and Hancock 2012; Fernández et al. 2012; Guerrero et al. 2013; Gunn 2008; Kawada-Matsuo et al. 2013; Nishino 2018). Specific TCS knockout strains have shown a significant decrease in the emergence of resistance. Apart from the TCSs, which are directly implicated in the emergence of drug resistance, some of them have been found to modulate the expression of drug efflux pumps, membrane transporter proteins that actively pump out the drugs from the bacterial cells leading to resistance. Most importantly, pathogens such as $K$. pneumoniae and A. baumannii possess several of these efflux systems that get signals from the TCS and lead to drug resistance (Laub and Goulian 2007; Stock et al. 2000).

Considering their role in different physiological processes of the bacterial cell including the development of drug resistance, the TCSs have been explored as novel drug targets (Bhagirath et al. 2019; Hirakawa et al. 2020; Tierney and Rather 2019; Worthington and Melander 2013). Several investigators have attempted to develop novel drugs by screening small molecules against the bacterial TCSs. A pioneering study in this regard was done in the Sperandio lab where a TCS QseCB in the E. coli was targeted as a part of their antivirulence strategy. QseCB is involved in the quorum-sensing pathway leading to the expression of virulence genes. High-throughput screening to search for a QseC kinase inhibitor led to the identification of the compound LED209, which specifically inhibits the autophosphorylation of the QseC kinase (Curtis et al. 2014; Rasko et al. 2008). An important TCS present among the firmicutes (low $\mathrm{G}+\mathrm{C}$ Gram-positive) group of bacteria known as WalKR, play an important role in the metabolism, stress response, virulence, and other regulatory pathways (Dubrac et al. 2008, 2007; Howden et al. 2011; Velikova et al. 2016). Because of its role in several physiological processes, WalKR has been used as a drug target and studies from the Utsumi group have shown promising results with the identification of a class of WalK inhibitors named walkmycin (Eguchi et al. 2011; Okada et al. 2010). These groups of compounds inhibit the formation of biofilm and the development of competence in Streptococcus mutans. Similarly, another WalK inhibitor named signermycin was also identified that targets the WalK dimerization (Watanabe et al. 2012).

Resistance against vancomycin, considered to be one of the most potent antibiotics, arises because of the VanSR TCS in the Enterococcus faecalis and E. faecium. Inhibitors of VanS kinases have been found that function by uncoupling the energy required for ATP synthesis. However, such inhibitors were not found to be suitable considering their negative impact on mitochondrial respiration. Nevertheless, such compounds serve as templates to develop more potent and specific inhibitors (Barrett et al. 1998; Macielag et al. 1998). In the case of $M$. tuberculosis, a TCS named DosRS (also called DevRS) contributes to its survival in the hypoxic 
conditions in the macrophages (Honaker et al. 2009; Park et al. 2003). Small molecule inhibitors have been identified against the DosR response regulator that can bind and inhibit its DNA binding property (Gupta et al. 2009; Mai et al. 2011). For the TCSs involved in polymyxin resistance pathways, studies have been conducted to find anti PhoPQ compounds. A compound called radicicol, a known Hsp90 inhibitor was found to inhibit the kinase activity of the PhoQ (Guarnieri et al. 2008). Similarly, virtual screening based on the putative PhoQ 3D structure of Shigella flexneri identified four different compounds that have potential anti-PhoQ kinase activities (Cai et al. 2011). These compounds exhibited a binding affinity for the cytoplasmic domain of the PhoQ inhibiting it. Interestingly, the compounds were also found to be inhibiting the $S$. flexneri to invade the HeLa cells in cell culture indicating their antivirulence characteristics (Cai et al. 2011).

Most of the drug candidates developed against the TCS work by blocking the signal transduction steps such as - inhibition of the sensor kinase activity, inhibition of the response regulator activity, signal sequestration, etc. (Hirakawa et al. 2020). All these studies show the potential of TCS-based antibiotic development, which needs to be seriously pursued.

3.3.2 Drug combination therapy: On many occasions, antibiotics are used in combinations for better treatment outcomes, to restrict the emergence of resistance, etc. In this regard, polymyxins seem to work synergistically with several other antibiotics. Carbapenems have been used in combination with polymyxins against the MDR Gram-negative pathogens resulting in impressive therapeutic outcomes (Clancy et al. 2013). The polymyxindoripenem combination was shown to be synergistic with enhanced bactericidal activity against the $A$. baumannii strains (Park et al. 2016). Against $P$. aeruginosa this combination has shown increased bactericidal activity including against the colistin heteroresistant subpopulations (Bergen et al. 2011; Deris et al. 2012). Interestingly, the polymyxin-doripenem combination also exhibited anti-biofilm activity in a dynamic biofilm model, as well as inhibited the emergence of colistin resistance highlighting the therapeutic potential of the combination (Lora-Tamayo et al. 2014).

Polymyxin-rifampicin combination showed very good effectiveness against MDR A. baumannii and KPC-producing K. pneumoniae (Biswas et al. 2012; Gaibani et al. 2014; Lagerback et al. 2016; Liang et al. 2011). Colistin in combination with meropenem and tigecycline has shown promising results against carbapenem-resistant $A$. baumannii strains (Liang et al. 2011; Sheng et al. 2011). Several studies have been conducted using novel combinations of polymyxin with other antibiotics including vancomycin, fosfomycin, aminoglycosides, daptomycin, doxycycline, etc. resulting in various efficacies and treatment outcomes (Nang et al. 2021).

In summary, the drug combination therapy could be a good alternative to polymyxin monotherapy considering the increased risk of the emergence of resistance. However, more rigorous studies are warranted to systematically explore the combination therapy approaches.

\section{Concluding remarks and future perspectives}

The rapid emergence of MDR pathogens has necessitated the urgent development of novel drugs and therapeutic interventions. As we have exhausted our arsenal of antibiotics, very few treatment options are available at the moment. The polymyxins, our last line antibiotics, therefore, must be used judiciously to prevent the onset of resistance. Recent studies using polymyxin have shown promising results clinically that have improved in designing a better dosing regimen. Also, the development of less toxic derivatives of polymyxins has reduced nephrotoxicity and neurotoxicity to a great extent. However, the mode of action of polymyxins at the molecular level is not yet fully understood, and at the same time, significant research effort must be put forth to develop an understanding of the resistance mechanism. These efforts would possibly further improve our treatment strategy in the future. The indiscriminate use of polymyxins must be curbed to preserve its usefulness at least till the time a better alternative drug emerges. The use of polymyxins as a growth supplement in animal farming must also be restricted which is responsible for the global spread of mobile colistin resistance $(\mathrm{mcr})$ genes. Meanwhile, investment in novel antibiotic drug development must be encouraged and pursued urgently to delay the arrival of the post-antibiotic era.

\section{Acknowledgements}

This work is supported by funding from the Science and Engineering Research Board (SERB), Govt. of India under the start-up research grant (SRG/2019/ 001703). Seed fund contributed by the Odisha State 
Higher Education Council (OSHEC), Government of Odisha, under the Odisha University Research and Innovation Incentivisation Plan (OURIIP-2019) is gratefully acknowledged.

\section{References}

Allen HK, Donato J, Wang HH, Cloud-Hansen KA, Davies $\mathrm{J}$, et al. 2010 Call of the wild: antibiotic resistance genes in natural environments. Nat. Rev. Microbiol. 8 251-259

Aquilini E, Merino S, Knirel YA, Regue M and Tomas JM 2014 Functional identification of Proteus mirabilis eptC gene encoding a core lipopolysaccharide phosphoethanolamine transferase. Int. J. Mol. Sci. 15 6689-6702

Arroyo LA, Herrera CM, Fernandez L, Hankins JV, Trent MS, et al. 2011 The pmrCAB operon mediates polymyxin resistance in Acinetobacter baumannii ATCC 17978 and clinical isolates through phosphoethanolamine modification of lipid A. Antimicrob. Agents Chemother. 55 3743-3751

Ayoub Moubareck C 2020 Polymyxins and Bacterial membranes: a review of antibacterial activity and mechanisms of resistance. Membranes 10181

Balaban NQ 2004 Bacterial persistence as a phenotypic switch. Science 305 1622-1625

Balaban NQ, Helaine S, Lewis K, Ackermann M, Aldridge B, et al. 2019 Definitions and guidelines for research on antibiotic persistence. Nat. Rev. Microbiol. 17 441-448

Barbosa C, Beardmore R, Schulenburg H and Jansen G 2018 Antibiotic combination efficacy (ACE) networks for a Pseudomonas aeruginosa model. PLOS Biol. 16 e2004356

Barrett JF, Goldschmidt RM, Lawrence LE, Foleno B, Chen $\mathrm{R}$, et al. 1998 Antibacterial agents that inhibit twocomponent signal transduction systems. Proc. Natl. Acad. Sci. USA 95 5317-5322

Bell G and MacLean C 2018 The search for 'evolutionproof' antibiotics. Trends Microbiol. 26 471-483

Bem AE, Velikova N, Pellicer MT, Pv Baarlen, Marina A, et al. 2015 Bacterial histidine kinases as novel antibacterial drug targets. ACS Chem. Biol. 10 213-224

Bengoechea JA and Skurnik M 2000 Temperature-regulated efflux pump/potassium antiporter system mediates resistance to cationic antimicrobial peptides in Yersinia. Mol. Microbiol. 37 67-80

Bergen PJ, Tsuji BT, Bulitta JB, Forrest A, Jacob J, et al. 2011 Synergistic killing of multidrug-resistant Pseudomonas aeruginosa at multiple inocula by colistin combined with doripenem in an in vitro pharmacokinetic/pharmacodynamic model. Antimicrob. Agents Chemother 55 5685-5695

Berglund NA, Piggot TJ, Jefferies D, Sessions RB, Bond PJ, et al. 2015 Interaction of the antimicrobial peptide polymyxin B1 with both membranes of E. coli: a molecular dynamics study. PLoS Comput. Biol. 11 e1004180

Bhagirath AY, Li Y, Patidar R, Yerex K, Ma X, et al. 2019 Two component regulatory systems and antibiotic resistance in gram-negative pathogens. Int. J. Mol. Sci. 201781

Bilecen K, Fong JC, Cheng A, Jones CJ, Zamorano-Sanchez $\mathrm{D}$, et al. 2015 Polymyxin B resistance and biofilm formation in Vibrio cholerae are controlled by the response regulator CarR. Infect. Immun. 83 1199-1209

Bina XR, Provenzano D, Nguyen N and Bina JE 2008 Vibrio cholerae RND family efflux systems are required for antimicrobial resistance, optimal virulence factor production, and colonization of the infant mouse small intestine. Infect. Immun. 76 3595-3605

Biswas S, Brunel JM, Dubus JC, Reynaud-Gaubert M and Rolain JM 2012 Colistin: an update on the antibiotic of the 21st century. Expert Rev. Anti Infect. Ther. 10 917-934

Blair JMA, Webber MA, Baylay AJ, Ogbolu DO and Piddock LJV 2015 Molecular mechanisms of antibiotic resistance. Nat. Rev. Microbiol. 13 42-51

Brennan-Krohn T, Pironti A and Kirby JE 2018 Synergistic Activity of Colistin-Containing Combinations against Colistin-Resistant Enterobacteriaceae. Antimicrob. Agents Chemother 62

Brochmann RP, Toft A, Ciofu O, Briales A, Kolpen M, et al. 2014 Bactericidal effect of colistin on planktonic Pseudomonas aeruginosa is independent of hydroxyl radical formation. Int J. Antimicrob. Agents 43 140-147

Cai X, Zhang J, Chen M, Wu Y, Wang X, et al. 2011 The effect of the potential PhoQ histidine kinase inhibitors on Shigella flexneri virulence. PLoS One 6 e 23100

Cannatelli A, Giani T, D'Andrea MM, Di Pilato V, Arena F, et al. $2014 \mathrm{MgrB}$ inactivation is a common mechanism of colistin resistance in KPC-producing Klebsiella pneumoniae of clinical origin. Antimicrob. Agents Chemother. $\mathbf{5 8}$ 5696-5703

Carabajal MA, Asquith CRM, Laitinen T, Tizzard GJ, Yim $\mathrm{L}$, et al. 2019 Quinazoline-based antivirulence compounds selectively target Salmonella $\mathrm{PhoP} / \mathrm{PhoQ}$ signal transduction system. Antimicrob. Agents Chemother. 64 e01744-01719

Carroll LM, Gaballa A, Guldimann C, Sullivan G, Henderson LO et al. 2019 Identification of novel mobilized colistin resistance gene mcr-9 in a multidrug-resistant, colistin-susceptible Salmonella enterica Serotype Typhimurium isolate. $m$ Bio $\mathbf{1 0}$

Chen HD and Groisman EA 2013 The biology of the PmrA/ PmrB two-component system: the major regulator of lipopolysaccharide modifications. Annu. Rev. Microbiol. $6783-112$

Cheng YH, Lin TL, Lin YT and Wang JT 2016 Amino acid substitutions of CrrB responsible for resistance to colistin through CrrC in Klebsiella pneumoniae. Antimicrob. Agents Chemother. 60 3709-3716 
Choi MJ and Ko KS 2014 Mutant prevention concentrations of colistin for Acinetobacter baumannii, Pseudomonas aeruginosa and Klebsiella pneumoniae clinical isolates. $J$. Antimicrob Chemother. 69 275-277

Clancy CJ, Chen L, Hong JH, Cheng S, Hao B, et al. 2013 Mutations of the ompK36 porin gene and promoter impact responses of sequence type 258, KPC-2-producing Klebsiella pneumoniae strains to doripenem and doripenemcolistin. Antimicrob. Agents Chemother. 57 5258-5265

Clausell A, Garcia-Subirats M, Pujol M, Busquets MA, Rabanal F, et al. 2007 Gram-negative outer and inner membrane models: insertion of cyclic cationic lipopeptides. J. Phys. Chem. B 111 551-563

Cortay JC and Cozzone AJ 1983a Accumulation of guanosine tetraphosphate induced by polymixin and gramicidin in Escherichia coli. Biochim. Biophys. Acta 755 467-473

Cortay JC and Cozzone AJ 1983b A study of bacterial response to polypeptide antibiotics. FEBS Lett. 157 307-310

Curtis MM, Russell R, Moreira CG, Adebesin AM, Wang C, et al. 2014 QseC inhibitors as an antivirulence approach for gram-negative pathogens. mBio 5 e02165-02114

Daugelavicius R, Bakiene E and Bamford DH 2000 Stages of polymyxin B interaction with the Escherichia coli cell envelope. Antimicrob. Agents Chemother. 44 2969-2978

David HL and Rastogi N 1985 Antibacterial action of colistin (polymyxin E) against Mycobacterium aurum. Antimicrob. Agents Chemother. 27 701-707

de Kraker ME, Stewardson AJ and Harbarth S 2016 Will 10 million people die a year due to antimicrobial resistance by 2050? PLoS Med 13 e1002184

De Oliveira DMP, Forde BM, Kidd TJ, Harris PNA, Schembri MA, et al. 2020 Antimicrobial resistance in ESKAPE pathogens. Clin. Microbiol. Rev. 33 e00181e1119

Defoirdt T 2018 Quorum-sensing systems as targets for antivirulence therapy. Trends Microbiol. 26 313-328

Depardieu F, Podglajen I, Leclercq R, Collatz E and Courvalin P 2007 Modes and modulations of antibiotic resistance gene expression. Clin. Microbiol. Rev. 20 $79-114$

Deris ZZ, Yu HH, Davis K, Soon RL, Jacob J, et al. 2012 The combination of colistin and doripenem is synergistic against Klebsiella pneumoniae at multiple inocula and suppresses colistin resistance in an in vitro pharmacokinetic/pharmacodynamic model. Antimicrob. Agents Chemother. 56 5103-5112

Doddangoudar VC, Boost MV, Tsang DN and O'Donoghue MM 2011 Tracking changes in the vraSR and graSR two component regulatory systems during the development and loss of vancomycin non-susceptibility in a clinical isolate. Clin. Microbiol. Infect. 17 1268-1272

Dubrac S, Bisicchia P, Devine KM and Msadek T 2008 A matter of life and death: cell wall homeostasis and the
WalKR (YycGF) essential signal transduction pathway. Mol. Microbiol. 70 1307-1322

Dubrac S, Boneca IG, Poupel O and Msadek T 2007 New insights into the WalK/WalR (YycG/YycF) essential signal transduction pathway reveal a major role in controlling cell wall metabolism and biofilm formation in Staphylococcus aureus. J. Bacteriol 189 8257-8269

Eguchi Y, Kubo N, Matsunaga H, Igarashi M and Utsumi R 2011 Development of an antivirulence drug against Streptococcus mutans: repression of biofilm formation, acid tolerance, and competence by a histidine kinase inhibitor, walkmycin C. Antimicrob. Agents Chemother. 55 1475-1484

El-Halfawy OM, Czarny TL, Flannagan RS, Day J, Bozelli JC, et al. 2020 Discovery of an antivirulence compound that reverses $\beta$-lactam resistance in MRSA. Nat. Chem. Biol. 16 143-149

Evans ME, Feola DJ and Rapp RP 1999 Polymyxin B sulfate and colistin: old antibiotics for emerging multiresistant gram-negative bacteria. Ann. Pharmacother. 33 960-967

Fehlner-Gardiner CC and Valvano MA 2002 Cloning and characterization of the Burkholderia vietnamiensis norM gene encoding a multi-drug efflux protein. FEMS Microbiol. Lett. 215 279-283

Fernández L, Álvarez-Ortega C, Wiegand I, Olivares J, Kocíncová D, et al. 2013 Characterization of the polymyxin B resistome of Pseudomonas aeruginosa. Antimicrob. Agents Chemother. 57 110-119

Fernández L, Gooderham WJ, Bains $\mathrm{M}$, McPhee JB, Wiegand I, et al. 2010 Adaptive resistance to the "last hope" antibiotics polymyxin B and colistin in Pseudomonas aeruginosa is mediated by the novel twocomponent regulatory system ParR-ParS. Antimicrob. Agents Chemother. 54 3372-3382

Fernandez L and Hancock REW 2012 Adaptive and mutational resistance: role of porins and efflux pumps in drug resistance. Clin. Microbiol. Rev. 25 661-681

Fernández L, Jenssen H, Bains M, Wiegand I, Gooderham WJ, et al. 2012 The two-component system CprRS senses cationic peptides and triggers adaptive resistance in Pseudomonas aeruginosa independently of ParRS. Antimicrob. Agents Chemother. 56 6212-6222

Fridman M, Williams GD, Muzamal U, Hunter H, Siu KW, et al. 2013 Two unique phosphorylation-driven signaling pathways crosstalk in Staphylococcus aureus to modulate the cell-wall charge: Stk1/Stp1 meets GraSR. Biochemistry 52 7975-7986

Gaibani P, Lombardo D, Lewis RE, Mercuri M, Bonora S, et al. 2014 In vitro activity and post-antibiotic effects of colistin in combination with other antimicrobials against colistin-resistant KPC-producing Klebsiella pneumoniae bloodstream isolates. J. Antimicrob. Chemother. 69 1856-1865

Gandra S, Alvarez-Uria G, Turner P, Joshi J, Limmathurotsakul D, et al. 2020 Antimicrobial resistance surveillance 
in low- and middle-income countries: progress and challenges in eight South Asian and Southeast Asian countries. Clin. Microbiol. Rev. 33 e00048-e119

Gonzalez-Avila LU, Loyola-Cruz MA, Hernández-Cortez C, Bello-López JM and Castro-Escarpulli G 2021 Colistin resistance in Aeromonas spp. Int J. Mol Sci 22

Gooderham WJ, Bains M, McPhee JB, Wiegand I and Hancock RE 2008 Induction by cationic antimicrobial peptides and involvement in intrinsic polymyxin and antimicrobial peptide resistance, biofilm formation, and swarming motility of PsrA in Pseudomonas aeruginosa. J. Bacteriol. 190 5624-5634

Groisman EA, Duprey A and Choi J 2021 How the PhoP/ PhoQ system controls virulence and $\mathrm{Mg}(2+)$ homeostasis: lessons in signal transduction, pathogenesis, physiology, and evolution. Microbiol. Mol. Biol. Rev.

Guarnieri MT, Zhang L, Shen J and Zhao R 2008 The Hsp90 inhibitor radicicol interacts with the ATP-binding pocket of bacterial sensor kinase PhoQ. J. Mol. Biol. 379 82-93

Guerrero P, Collao B, Alvarez R, Salinas H, Morales EH, et al. 2013 Salmonella enterica serovar Typhimurium BaeSR two-component system positively regulates sodA in response to ciprofloxacin. Microbiology 159 2049-2057

Gunn JS 2008 The Salmonella PmrAB regulon: lipopolysaccharide modifications, antimicrobial peptide resistance and more. Trends Microbiol. 16 284-290

Gunn JS, Belden WJ and Miller SI 1998a Identification of PhoP-PhoQ activated genes within a duplicated region of the Salmonella typhimurium chromosome. Microb. Pathog. 25 77-90

Gunn JS, Lim KB, Krueger J, Kim K, Guo L, et al. 1998b PmrA-PmrB-regulated genes necessary for 4-aminoarabinose lipid A modification and polymyxin resistance. Mol. Microbiol. 27 1171-1182

Gupta RK, Thakur TS, Desiraju GR and Tyagi JS 2009 Structure-based design of DevR inhibitor active against nonreplicating Mycobacterium tuberculosis. J. Med. Chem. 52 6324-6334

Gutu AD, Sgambati N, Strasbourger P, Brannon MK, Jacobs MA, et al. 2013 Polymyxin resistance of Pseudomonas aeruginosa pho $Q$ mutants is dependent on additional twocomponent regulatory systems. Antimicrob. Agents Chemother. 57 2204-2215

Han ML, Velkov T, Zhu Y, Roberts KD, Le Brun AP, et al. 2018 Polymyxin-induced lipid A deacylation in Pseudomonas aeruginosa perturbs polymyxin penetration and confers high-level resistance. ACS Chem. Biol. 13 121-130

Hancock RE 1984 Alterations in outer membrane permeability. Annu. Rev. Microbiol. 38 237-264

Hancock RE 1997 Peptide antibiotics. Lancet 349 418-422

Hancock RE and Chapple DS 1999 Peptide antibiotics. Antimicrob. Agents Chemother. 43 1317-1323

Harms A, Maisonneuve E and Gerdes K 2016 Mechanisms of bacterial persistence during stress and antibiotic exposure. Science 354 aaf 4268
Herrera CM, Crofts AA, Henderson JC, Pingali SC, Davies BW et al. 2014 The Vibrio cholerae VprA-VprB twocomponent system controls virulence through endotoxin modification. mBio $\mathbf{5}$

Hirakawa H, Kurushima J, Hashimoto Y and Tomita H 2020 Progress overview of bacterial two-component regulatory systems as potential targets for antimicrobial chemotherapy. Antibiotics 9635

Hiron A, Falord M, Valle J, Debarbouille M and Msadek T 2011 Bacitracin and nisin resistance in Staphylococcus aureus: a novel pathway involving the BraS/BraR twocomponent system (SA2417/SA2418) and both the BraD/ $\mathrm{BraE}$ and $\mathrm{VraD} / \mathrm{VraE} \mathrm{ABC}$ transporters. Mol. Microbiol. 81 602-622

Honaker RW, Leistikow RL, Bartek IL and Voskuil MI 2009 Unique roles of DosT and DosS in DosR regulon induction and Mycobacterium tuberculosis dormancy. Infect Immun. 77 3258-3263

Howden BP, McEvoy CR, Allen DL, Chua K, Gao W, et al. 2011 Evolution of multidrug resistance during Staphylococcus aureus infection involves mutation of the essential two component regulator WalKR. PLoS Pathog 7 e1002359

Huang J, Li C, Song J, Velkov T, Wang L, et al. 2020 Regulating polymyxin resistance in Gram-negative bacteria: roles of two-component systems PhoPQ and PmrAB. Future Microbiology 15 445-459

Imlay JA 2015 Diagnosing oxidative stress in bacteria: not as easy as you might think. Curr. Opin. Microbiol. 24 124-131

Jiang SS, Lin TY, Wang WB, Liu MC, Hsueh PR, et al. 2010 Characterization of UDP-glucose dehydrogenase and UDP-glucose pyrophosphorylase mutants of Proteus mirabilis: defectiveness in polymyxin B resistance, swarming, and virulence. Antimicrob. Agents Chemother. 54 2000-2009

Kawada-Matsuo M, Oogai Y, Zendo T, Nagao J, Shibata Y, et al. 2013 Involvement of the novel two-component NsrRS and LcrRS systems in distinct resistance pathways against nisin A and nukacin ISK-1 in Streptococcus mutans. Appl. Environ. Microbiol. 79 4751-4755

Knight GM, Glover RE, McQuaid CF, Olaru ID, Gallandat $\mathrm{K}$ et al. 2021 Antimicrobial resistance and COVID-19: Intersections and implications. Elife $\mathbf{1 0}$

Kohanski MA, Dwyer DJ, Hayete B, Lawrence CA and Collins JJ 2007 A common mechanism of cellular death induced by bactericidal antibiotics. Cell $130797-810$

Lagerback P, Khine WW, Giske CG and Tangden T 2016 Evaluation of antibacterial activities of colistin, rifampicin and meropenem combinations against NDM-1-producing Klebsiella pneumoniae in $24 \mathrm{~h}$ in vitro time-kill experiments. J. Antimicrob. Chemother. 71 2321-2325

Lai CC, Chen SY, Ko WC and Hsueh PR 2021 Increased antimicrobial resistance during the COVID-19 pandemic. Int J. Antimicrob. Agents 57106324 
Landman D, Georgescu C, Martin DA and Quale J 2008 Polymyxins revisited. Clin. Microbiol. Rev. 21 449-465

Laub MT and Goulian M 2007 Specificity in two-component signal transduction pathways. Annu. Rev. Genet. 41 121-145

Liang W, Liu XF, Huang J, Zhu DM, Li J, et al. 2011 Activities of colistin- and minocycline-based combinations against extensive drug resistant Acinetobacter baumannii isolates from intensive care unit patients. BMC Infect. Dis. 11109

Limmathurotsakul D, Dunachie S, Fukuda K, Feasey NA, Okeke IN, et al. 2019 Improving the estimation of the global burden of antimicrobial resistant infections. Lancet Infect. Dis. 19 e392-e398

Lin QY, Tsai YL, Liu MC, Lin WC, Hsueh PR, et al. 2014 Serratia marcescens arn, a PhoP-regulated locus necessary for polymyxin B resistance. Antimicrob. Agents Chemother. 58 5181-5190

Lin Y-W, Han M-L, Zhao J, Zhu Y, Rao G, et al. 2019 Synergistic Combination of polymyxin B and enrofloxacin induced metabolic perturbations in extensive drug-resistant Pseudomonas aeruginosa. Front. Pharmacol. 101146

Liu J, Xiao G, Zhou W, Yang J, Wang Y, et al. 2021 Various novel colistin resistance mechanisms interact to facilitate adaptation of Aeromonas hydrophila to complex colistin environments. Antimicrob. Agents Chemother. 65 e0007121

Liu YY, Wang Y, Walsh TR, Yi LX, Zhang R, et al. 2016 Emergence of plasmid-mediated colistin resistance mechanism MCR-1 in animals and human beings in China: a microbiological and molecular biological study. Lancet Infect. Dis. 16 161-168

Llobet E, Tomas JM and Bengoechea JA 2008 Capsule polysaccharide is a bacterial decoy for antimicrobial peptides. Microbiology 154 3877-3886

Lora-Tamayo J, Murillo O, Bergen PJ, Nation RL, Poudyal A, et al. 2014 Activity of colistin combined with doripenem at clinically relevant concentrations against multidrug-resistant Pseudomonas aeruginosa in an in vitro dynamic biofilm model. J. Antimicrob. Chemother. 69 2434-2442

Macfarlane ELA, Kwasnicka A and Hancock REW 2000 Role of Pseudomonas aeruginosa PhoP-phoQ in resistance to antimicrobial cationic peptides and aminoglycosides. Microbiology 146 2543-2554

Macielag MJ, Demers JP, Fraga-Spano SA, Hlasta DJ, Johnson SG, et al. 1998 Substituted salicylanilides as inhibitors of two-component regulatory systems in bacteria. J. Med. Chem. 41 2939-2945

MacNair CR, Stokes JM, Carfrae LA, Fiebig-Comyn AA, Coombes BK, et al. 2018 Overcoming mcr-1 mediated colistin resistance with colistin in combination with other antibiotics. Nat. Commun. 9458

Mai D, Jones J, Rodgers JW, Hartman JLT, Kutsch O, et al. 2011 A screen to identify small molecule inhibitors of protein-protein interactions in mycobacteria. Assay Drug Dev. Technol. 9 299-310

Mathur J and Waldor MK 2004 The Vibrio cholerae ToxRregulated porin OmpU confers resistance to antimicrobial peptides. Infect. Immun. 72 3577-3583

McConville TH, Annavajhala MK, Giddins MJ, Macesic N, Herrera CM, et al. 2020 CrrB positively regulates highlevel polymyxin resistance and virulence in Klebsiella pneumoniae. Cell Rep 33108313

McCoy LS, Roberts KD, Nation RL, Thompson PE, Velkov $\mathrm{T}$, et al. 2013 Polymyxins and analogues bind to ribosomal RNA and interfere with eukaryotic translation in vitro. Chembiochem 14 2083-2086

McPhee JB, Lewenza S and Hancock RE 2003 Cationic antimicrobial peptides activate a two-component regulatory system, PmrA-PmrB, that regulates resistance to polymyxin $\mathrm{B}$ and cationic antimicrobial peptides in Pseudomonas aeruginosa. Mol Microbiol 50 205-217

Mmatli M, Mbelle NM, Maningi NE and OseiSekyere J 2020 Emerging transcriptional and genomic mechanisms mediating carbapenem and polymyxin resistance in Enterobacteriaceae: a systematic review of current reports. eSystems 5 e00783-20

Moffatt JH, Harper M, Adler B, Nation RL, Li J, et al. 2011 Insertion sequence ISAba11 is involved in colistin resistance and loss of lipopolysaccharide in Acinetobacter baumannii. Antimicrob. Agents Chemother. 55 3022-3024

Moffatt JH, Harper M and Boyce JD 2019 Mechanisms of polymyxin resistance. Adv. Exp. Med. Biol. 1145 55-71

Moffatt JH, Harper M, Harrison P, Hale JD, Vinogradov E, et al. 2010 Colistin resistance in Acinetobacter baumannii is mediated by complete loss of lipopolysaccharide production. Antimicrob. Agents Chemother. 54 4971-4977

Mogi T, Matsushita K, Murase Y, Kawahara K, Miyoshi H, et al. 2009 Identification of new inhibitors for alternative NADH dehydrogenase (NDH-II). FEMS Microbiol. Lett. 291 157-161

Moon K and Gottesman S 2009 A PhoQ/P-regulated small RNA regulates sensitivity of Escherichia coli to antimicrobial peptides. Mol. Microbiol. 74 1314-1330

Mortensen NP, Fowlkes JD, Sullivan CJ, Allison DP, Larsen NB, et al. 2009 Effects of colistin on surface ultrastructure and nanomechanics of Pseudomonas aeruginosa cells. Langmuir 25 3728-3733

Mouslim C and Groisman EA 2003 Control of the Salmonella ugd gene by three two-component regulatory systems. Mol. Microbiol. 47 335-344

Nakajima K and Kawamata J 1966 Studies on the mechanism of action of colistin. IV. Activation of "latent" ribonuclease in Escherichia coli by colistin. Biken $J 9$ $115-123$

Nang SC, Azad MAK, Velkov T, Zhou QT and Li J 2021 Rescuing the last-line polymyxins: achievements and challenges. Pharmacol. Rev. 73 679-728 
Nation RL, Velkov T and Li J 2014 Colistin and polymyxin B: peas in a pod, or chalk and cheese? Clin. Infect. Dis. 59 88-94

Newton BA 1956 The properties and mode of action of the polymyxins. Bacteriol. Rev. 20 14-27

Nguyen HT, Wolff KA, Cartabuke RH, Ogwang S and Nguyen L 2010 A lipoprotein modulates activity of the MtrAB two-component system to provide intrinsic multidrug resistance, cytokinetic control and cell wall homeostasis in Mycobacterium. Mol. Microbiol. 76 348-364

Nishino K 2018 Regulation of the expression of bacterial multidrug exporters by two-component signal transduction systems; in Bacterial multidrug exporters (eds) Yamaguchi A and Nishino K (New York: Springer) 239-251

O’Neill J 2016 Tackling drug-resistant infections globally: final report and recommendations. Review on Antimicrobial Resistance, May 2016

Ogawara H 2021 Possible drugs for the treatment of bacterial infections in the future: anti-virulence drugs. $J$. Antibiot. 74 24-41

Okada A, Igarashi M, Okajima T, Kinoshita N, Umekita M, et al. 2010 Walkmycin B targets WalK (YycG), a histidine kinase essential for bacterial cell growth. $J$. Antibiot. 63 89-94

Olaitan AO, Morand S and Rolain JM 2014 Mechanisms of polymyxin resistance: acquired and intrinsic resistance in bacteria. Front. Microbiol. 5643

Olsson A, Wistrand-Yuen P, Nielsen EI, Friberg LE, Sandegren L, et al. 2020 Efficacy of antibiotic combinations against multidrug-resistant Pseudomonas aeruginosa in automated time-lapse microscopy and static timekill experiments. Antimicrob. Agents Chemother. 64 e02111-02119

Osborn MJ, Gander JE, Parisi E and Carson J 1972 Mechanism of assembly of the outer membrane of Salmonella typhimurium. Isolation and characterization of cytoplasmic and outer membrane. J. Biol. Chem. 247 3962-3972

Padilla E, Llobet E, Domenech-Sanchez A, MartinezMartinez L, Bengoechea JA, et al. 2010 Klebsiella pneumoniae AcrAB efflux pump contributes to antimicrobial resistance and virulence. Antimicrob. Agents Chemother. 54 177-183

Park GC, Choi JA, Jang SJ, Jeong SH, Kim CM, et al. 2016 In vitro interactions of antibiotic combinations of colistin, tigecycline, and doripenem against extensively drugresistant and multidrug-resistant Acinetobacter baumannii. Ann. Lab. Med. 36 124-130

Park HD, Guinn KM, Harrell MI, Liao R, Voskuil MI, et al. $2003 \mathrm{Rv} 3133 \mathrm{c} / \mathrm{dos} \mathrm{R}$ is a transcription factor that mediates the hypoxic response of Mycobacterium tuberculosis. Mol. Microbiol. 48 833-843

Poirel L, Jayol A, Bontron S, Villegas MV, Ozdamar M, et al. 2015 The $\operatorname{mgr} B$ gene as a key target for acquired resistance to colistin in Klebsiella pneumoniae. J. Antimicrob. Chemother. 70 75-80

Poirel L, Jayol A and Nordmann P 2017a Polymyxins: antibacterial activity, susceptibility testing, and resistance mechanisms encoded by plasmids or chromosomes. Clin. Microbiol. Rev. 30 557-596

Poirel L, Kieffer N and Nordmann P 2017b In vitro study of ISApl1-mediated mobilization of the colistin resistance gene mor-1. Antimicrob Agents Chemother 61

Raetz CR, Reynolds CM, Trent MS and Bishop RE 2007 Lipid A modification systems in gram-negative bacteria. Annu. Rev. Biochem. 76 295-329

Rasko DA, Moreira CG, de Li R, Reading NC, Ritchie JM, et al. 2008 Targeting QseC signaling and virulence for antibiotic development. Science 321 1078-1080

Rawson TM, Ming D, Ahmad R, Moore LSP and Holmes AH 2020a Antimicrobial use, drug-resistant infections and COVID-19. Nat. Rev. Microbiol. 18 409-410

Rawson TM, Moore LSP, Castro-Sanchez E, Charani E, Davies F, et al. 2020b COVID-19 and the potential longterm impact on antimicrobial resistance. J. Antimicrob. Chemother. 75 1681-1684

Rosch JW, Hsu FF and Caparon MG 2007 Anionic lipids enriched at the ExPortal of Streptococcus pyogenes. J. Bacteriol. 189 801-806

Sabnis A, Hagart KL, Klöckner A, Becce M, Evans LE, et al. 2021 Colistin kills bacteria by targeting lipopolysaccharide in the cytoplasmic membrane. Elife 10 e65836

Schrader SM, Vaubourgeix J and Nathan C 2020 Biology of antimicrobial resistance and approaches to combat it. Sci. Translat. Med. 12 eaaz6992

Sheng WH, Wang JT, Li SY, Lin YC, Cheng A, et al. 2011 Comparative in vitro antimicrobial susceptibilities and synergistic activities of antimicrobial combinations against carbapenem-resistant Acinetobacter species: Acinetobacter baumannii versus Acinetobacter genospecies 3 and 13TU. Diagn. Microbiol. Infect. Dis. 70 380-386

Simpson BW and Trent MS 2019 Pushing the envelope: LPS modifications and their consequences. Nat. Rev. Microbiol. 17 403-416

Spindler EC, Hale JD, Giddings TH Jr, Hancock RE and Gill RT 2011 Deciphering the mode of action of the synthetic antimicrobial peptide Bac8c. Antimicrob. Agents Chemother. 55 1706-1716

Srinivasan VB, Singh BB, Priyadarshi N, Chauhan NK and Rajamohan G 2014 Role of novel multidrug efflux pump involved in drug resistance in Klebsiella pneumoniae. PLoS One 9 e96288

Stock AM, Robinson VL and Goudreau PN 2000 Twocomponent signal transduction. Annu. Rev. Biochem. 69 $183-215$

Storm DR, Rosenthal KS and Swanson PE 1977 Polymyxin and related peptide antibiotics. Annu. Rev. Biochem. 46 $723-763$ 
Teuber M 1967 Precipitation of ribosomes from E. coli B by polymyxin B. Naturwissenschaften $\mathbf{5 4} 71$

Tierney AR and Rather PN 2019 Roles of two-component regulatory systems in antibiotic resistance. Future Microbiol. 14 533-552

Tochikubo K, Yasuda Y and Kozuka S 1986 Decreased particulate NADH oxidase activity in Bacillus subtilis spores after polymyxin B treatment. J. Gen. Microbiol. 132 277-287

Trent MS, Ribeiro AA, Lin S, Cotter RJ and Raetz CR 2001 An inner membrane enzyme in Salmonella and Escherichia coli that transfers 4-amino-4-deoxy-L-arabinose to lipid A: induction on polymyxin-resistant mutants and role of a novel lipid-linked donor. J. Biol. Chem. 276 43122-43131

Trimble MJ, Mlynarcik P, Kolar M and Hancock RE 2016 Polymyxin: alternative mechanisms of action and resistance. Cold Spring Harb Perspect Med 6

Tsai MH, Wu SR, Lee HY, Chen CL, Lin TY, et al. 2012 Recognition of mechanisms involved in bile resistance important to halting antimicrobial resistance in nontyphoidal Salmonella. Int. J. Antimicrob. Agents $\mathbf{4 0}$ 151-157

Tzeng YL, Ambrose KD, Zughaier S, Zhou X, Miller YK, et al. 2005 Cationic antimicrobial peptide resistance in Neisseria meningitidis. J. Bacteriol. 187 5387-5396

Vega LA and Caparon MG 2012 Cationic antimicrobial peptides disrupt the Streptococcus pyogenes ExPortal. Mol. Microbiol. 85 1119-1132

Velikova N, Fulle S, Manso AS, Mechkarska M, Finn P, et al. 2016 Putative histidine kinase inhibitors with antibacterial effect against multi-drug resistant clinical isolates identified by in vitro and in silico screens. Sci. Rep. 626085

Velkov T, Thompson PE, Nation RL and Li J 2010 Structure-activity relationships of polymyxin antibiotics. J. Med. Chem. 53 1898-1916

Corresponding editor: SudHA BHATTACHARYA
Warner DM and Levy SB 2010 Different effects of transcriptional regulators MarA, SoxS and Rob on susceptibility of Escherichia coli to cationic antimicrobial peptides (CAMPs): Rob-dependent CAMP induction of the marRAB operon. Microbiology 156 570-578

Watanabe T, Igarashi M, Okajima T, Ishii E, Kino $\mathrm{H}$, et al. 2012 Isolation and characterization of signermycin B, an antibiotic that targets the dimerization domain of histidine kinase WalK. Antimicrob. Agents Chemother. 56 3657-3663

WHO WHOJG 2015 Antimicrobial resistance draft global action plan on antimicrobial resistance.

Worthington RJ and Melander C 2013 Combination approaches to combat multidrug-resistant bacteria. Trends Biotechnol. 31 177-184

Wright MS, Suzuki Y, Jones MB, Marshall SH, Rudin SD, et al. 2015 Genomic and transcriptomic analyses of colistin-resistant clinical isolates of Klebsiella pneumoniae reveal multiple pathways of resistance. Antimicrob. Agents Chemother. 59 536-543

Yeung AT, Bains M and Hancock RE 2011 The sensor kinase $\mathrm{CbrA}$ is a global regulator that modulates metabolism, virulence, and antibiotic resistance in Pseudomonas aeruginosa. J. Bacteriol. 193 918-931

Yin J, Meng Q, Cheng D, Fu J, Luo Q, et al. 2020 Mechanisms of bactericidal action and resistance of polymyxins for Gram-positive bacteria. Appl. Microbiol. Biotechnol. 104 3771-3780

Yu Z, Qin W, Lin J, Fang S and Qiu J 2015 Antibacterial Mechanisms of Polymyxin and Bacterial Resistance. BioMed. Res. Int. 2015 1-11

Zhang L, Dhillon P, Yan H, Farmer S and Hancock RE 2000 Interactions of bacterial cationic peptide antibiotics with outer and cytoplasmic membranes of Pseudomonas aeruginosa. Antimicrob. Agents Chemother. $\mathbf{4 4}$ $3317-3321$ 